\title{
THE EQUILIBRIUM STATES \\ FOR SEMIGROUPS OF RATIONAL MAPS
}

\author{
HIROKI SUMI AND MARIUSZ URBAŃSKI
}

\begin{abstract}
We consider the dynamics of skew product maps associated with finitely generated semigroups of rational maps on the Riemann sphere. We show that under some conditions on the dynamics and the potential function $\psi$, there exists a unique equilibrium state for $\psi$ and a unique $\exp (\mathrm{P}(\psi)-\psi)$-conformal measure, where $\mathrm{P}(\psi)$ denotes the topological pressure of $\psi$.
\end{abstract}

\section{Introduction}

In this paper, we frequently use the notation from [13]. A "rational semigroup" $G$ is a semigroup generated by a family of non-constant rational maps $g: \overline{\mathbb{C}} \rightarrow \overline{\mathbb{C}}$, where $\overline{\mathbb{C}}$ denotes the Riemann sphere, with the semigroup operation being functional composition. For a rational semigroup $G$, we set $F(G):=\{z \in \overline{\mathbb{C}} \mid G$ is normal in a neighborhood of $z\}$ and $J(G):=\overline{\mathbb{C}} \backslash F(G) . F(G)$ is called the Fatou set of $G$ and $J(G)$ is called the Julia set of $G$. If $G$ is generated by a family $\left\{f_{i}\right\}_{i}$, then we write $G=\left\langle f_{1}, f_{2}, \ldots\right\rangle$.

The research on the dynamics of rational semigroups was initiated by Hinkkanen and Martin ([8]), who were interested in the role of the dynamics of polynomial semigroups while studying various one-complexdimensional moduli spaces for discrete groups, and by F. Ren's group ([21]), who studied such semigroups from the perspective of random complex dynamics. For further studies on the dynamics of rational semigroups, see $[13,14,15,16,17,18,12,19]$.

The theory of the dynamics of rational semigroups is deeply related to that of the fractal geometry. In fact, if $G=\left\langle f_{1}, \ldots, f_{s}\right\rangle$ is a finitely

Key words and phrases. rational semigroup, skew product, equilibrium state.

Date: August 19, 2008. To appear in Monatsh. Math. 2000 Mathematics Subject Classification. Primary: 37F10, Secondary: 37A35.

The research of the second author was supported in part by the NSF Grant DMS 0400481. The first author thanks University of North Texas for kind hospitality, during his stay there. 
generated rational semigroup, then the Julia set $J(G)$ of $G$ has the "backward self-similarity", i.e.,

$$
J(G)=f_{1}^{-1}(J(G)) \cup \cdots \cup f_{s}^{-1}(J(G))
$$

(See [13]). Hence, the behavior of the (backward) dynamics of finitely generated rational semigroups can be regarded as that of the "backward iterated function systems." For example, the Sierpiński gasket can be regarded as the Julia set of a rational semigroup.

The research on the dynamics of rational semigroups is also directly related to that on the random dynamics of holomorphic maps. The first study on the random dynamics of holomorphic maps was by Fornaess and Sibony ([7]), and much research has followed. (See $[1,2,3,4]$.) In fact, it is very natural and important to combine both the theory of rational semigroups and that of random complex dynamics (see [13, 14, $16,17,18])$.

We use throughout spherical derivatives and, for each meromorphic function $\varphi$, we denote by $\left|\varphi^{\prime}(z)\right|$ the norm of the derivative with respect to the spherical metric. We denote by $C V(\varphi)$ the set of critical values of $\varphi$. The symbol $A$ is used to denote the spherical area. We set $\kappa=$ $A(B(0,1)) / 4$. Obviously, there exists a constant $C_{s a} \geq 1$ such that for each $0<R \leq \operatorname{diam}(\bar{C}) / 2, C_{s a}^{-1} \leq A(B(z, R)) / \kappa(2 R)^{2} \leq C_{s a}$.

Let $G=\left\langle f_{1}, \ldots, f_{s}\right\rangle$ be a finitely generated rational semigroup. Then, we use the following notation. Let $\Sigma_{s}:=\{1, \ldots, s\}^{\mathbb{N}}$ be the space of one-sided sequences of $s$-symbols endowed with the product topology. This is a compact metric space. Let $f: \Sigma_{s} \times \overline{\mathbb{C}} \rightarrow \Sigma_{s} \times \overline{\mathbb{C}}$ be the skew product map associated with $\left\{f_{1}, \ldots, f_{s}\right\}$ given by the formula, $f(\omega, z)=\left(\sigma(\omega), f_{\omega_{1}}(z)\right)$, where $(\omega, z) \in \Sigma_{s} \times \overline{\mathbb{C}}, \omega=\left(\omega_{1}, \omega_{2}, \ldots\right)$, and $\sigma: \Sigma_{s} \rightarrow \Sigma_{s}$ denotes the shift map. We denote by $\pi_{1}: \Sigma_{s} \times \overline{\mathbb{C}} \rightarrow \Sigma_{s}$ the projection onto $\Sigma_{s}$ and $\pi_{2}: \Sigma_{s} \times \overline{\mathbb{C}} \rightarrow \overline{\mathbb{C}}$ the projection onto $\overline{\mathbb{C}}$. That is, $\pi_{1}(\omega, z)=\omega$ and $\pi_{2}(\omega, z)=z$. Under the canonical identification $\pi_{1}^{-1}\{\omega\} \cong \overline{\mathbb{C}}$, each fiber $\pi_{1}^{-1}\{\omega\}$ is a Riemann surface which is isomorphic to $\overline{\mathbb{C}}$.

Let $\operatorname{Crit}(f):=\bigcup_{\omega \in \Sigma_{s}}\left\{v \in \pi_{1}^{-1}\{\omega\} \mid v\right.$ is a critical point of $\left.f\right|_{\pi_{1}^{-1}\{\omega\}} \rightarrow$ $\left.\pi_{1}^{-1}\{\sigma(\omega)\}\right\}\left(\subset \Sigma_{s} \times \overline{\mathbb{C}}\right)$ be the set of critical points of $f$. For each $n \in \mathbb{N}$ and $(\omega, z) \in \Sigma_{s} \times \overline{\mathbb{C}}$, we set $\left(f^{n}\right)^{\prime}(\omega, z):=\left(f_{\omega_{n}} \circ \cdots \circ f_{\omega_{1}}\right)^{\prime}(z)$.

For each $\omega \in \Sigma_{s}$ we define $J_{\omega}:=\left\{z \in \overline{\mathbb{C}} \mid\left\{f_{\omega_{n}} \circ \cdots \circ f_{\omega_{1}}\right\}_{n \in \mathbb{N}}\right.$ is not normal in any neighborhood of $\left.z\right\}$ and we then set

$$
J(f):=\overline{\cup_{w \in \Sigma_{s}}\{\omega\} \times J_{\omega}},
$$


where the closure is taken in the product space $\Sigma_{s} \times \overline{\mathbb{C}}$. By definition, $J(f)$ is compact. Furthermore, by Proposition 3.2 in [13], $J(f)$ is completely invariant under $f, f$ is an open map on $J(f),(f, J(f))$ is topologically exact under a mild condition, and $J(f)$ is equal to the closure of the set of repelling periodic points of $f$ provided that $\sharp J(G) \geq 3$, where we say that a periodic point $(\omega, z)$ of $f$ with period $n$ is repelling if $\left|\left(f^{n}\right)^{\prime}(\omega, z)\right|>1$. Furthermore, $\pi_{2}(J(f))=J(G)$. We set

$$
e_{j}:=\operatorname{deg}\left(f_{j}\right) \text { and } d:=\max _{j=1, \ldots, s}\left(2 e_{j}-2\right) .
$$

Throughout the paper, we assume the following.

(E1) There exists an element $g \in G$ with $\operatorname{deg}(g) \geq 2$. Furthermore, for the semigroup $H:=\left\{h^{-1} \mid h \in \operatorname{Aut} \overline{\mathscr{C}} \cap G\right\}$, we have $J(G) \subset$ $F(H)$. (If $H$ is empty, we put $F(H):=\overline{\mathbb{C}}$.)

(E2) $\sharp($ Crit $(f) \cap J(f))<\infty$

(E3) There is no super attracting cycle of $f$ in $J(f)$. That is, if $(\omega, z) \in$ $J(f)$ is a periodic point with period $n$, then $\left(f^{n}\right)^{\prime}(\omega, z) \neq 0$.

Any finitely generated rational semigroup $G=\left\langle f_{1}, \ldots, f_{s}\right\rangle$ satisfying all the conditions (E1)-(E3) will be called an E-semigroup of rational maps.

\section{Examples.}

- If $g$ is a rational map with $\operatorname{deg}(g) \geq 2$, then $\langle g\rangle$ is an E-semigroup of rational maps.

- Let $G=\left\langle f_{1}, \ldots, f_{s}\right\rangle$ be a finitely generated rational semigroup such that $\operatorname{deg}\left(f_{j}\right) \geq 2$ for each $j=1, \ldots, s$. If $\mathrm{CV}\left(f_{j}\right) \cap J(G)=\emptyset$ for each $j=1, \ldots, s$, then $G$ is an E-semigroup of rational maps.

- Let $g_{1}$ and $g_{2}$ be two polynomials with $\operatorname{deg}\left(g_{j}\right) \geq 2(j=1,2)$. Suppose that

$$
\overline{\cup_{n=0}^{\infty} g_{1}^{n}\left(C V\left(g_{1}\right)\right)} \subset A_{\infty}\left(g_{2}\right) \text { and } \overline{\cup_{n=0}^{\infty} g_{2}^{n}\left(C V\left(g_{2}\right)\right)} \subset A_{\infty}\left(g_{1}\right),
$$

where $A_{\infty}(\cdot)$ denotes the basin of infinity. Let $m \in \mathbb{N}$ be a sufficiently large number so that

$$
g_{1}^{m}\left(\overline{\cup_{n=0}^{\infty} g_{2}^{n}\left(C V\left(g_{2}\right)\right)}\right) \subset U_{\infty} \text { and } g_{2}^{m}\left(\overline{\cup_{n=0}^{\infty} g_{1}^{n}\left(C V\left(g_{1}\right)\right)}\right) \subset U_{\infty}
$$

where $U_{\infty}$ denotes the connected component of $F\left(\left\langle g_{1}, g_{2}\right\rangle\right)$ with $\infty \in U_{\infty}$. Then, the semigroup $\left\langle g_{1}^{m}, g_{2}^{m}\right\rangle$ is an E-semigroup of rational maps.

The dynamics of the skew product map $f: \Sigma_{s} \times \overline{\mathbb{C}} \rightarrow \Sigma_{s} \times \overline{\mathbb{C}}$ is directly related to the dynamics of the semigroup $G=\left\langle f_{1}, \ldots, f_{s}\right\rangle$. For example, 
we use the dynamics of $f$ to analyze the dimension of Julia set $J(G)$ of $G$ (see $[13,14,15,16,19])$.

Our main concern in this paper is the existence and uniqueness of equilibrium states for all $E$-semigroups of rational maps and a large class of Hölder continuous potentials. We do not assume any kind of expanding property. If $T: X \rightarrow X$ is a continuous mapping of a compact metric space $X$, then one can define the topological pressure $\mathrm{P}(g)$ for every real-valued continuous function $g$ on $X$ (see [20] for instance) in purely topological terms. The link with measurable dynamics is given by the Variational Principle (see [20] again) saying that the topological pressure $\mathrm{P}(g)$ is equal to the supremum of all numbers $\mathrm{h}_{\mu}(T)+\int g d \mu$, $\mu$ being Borel probability $T$-invariant measures on $X$. Any invariant measure $\mu$ satisfying equality

$$
\mathrm{P}(g)=\mathrm{h}_{\mu}(T)+\int g d \mu
$$

is called an equilibrium state. All equilibrium states have a profound physical meaning (see [11]), their existence as well as uniqueness is of primary importance and is in general not clear at all. A general sufficient condition for the existence is expansiveness, a weaker one, holding for all rational maps on the sphere (see [9]), is asymptotic $h$-expansiveness. This covers the case of hyperbolic maps studied in [15]. Our goal in this paper is to do both, existence and uniqueness of equilibrium states, for E-semigroup of rational maps and a large class of Hölder continuous potentials. Thus, the main purpose of this paper is to prove the following result.

Theorem 1.1. (Main result) Let $G=\left\langle f_{1}, \ldots, f_{s}\right\rangle$ be an E-semigroup of rational maps. Let $f: \Sigma_{s} \times \overline{\mathbb{C}} \rightarrow \Sigma_{s} \times \overline{\mathbb{C}}$ be the skew product map associated with $\left\{f_{1}, \ldots, f_{s}\right\}$. Let $\psi: J(f) \rightarrow \mathbb{R}$ be a Hölder continuous function. Moreover, let $P^{p}(\psi)$ be the pointwise pressure of $\psi$ with respect to the dynamics of $f: J(f) \rightarrow J(f)$ (see the definition (4.2)). Suppose that $\mathrm{P}^{p}(\psi)>\sup (\psi)+\log s$. Then, all of the following statements hold.

(1) Regarding the dynamics of $f: J(f) \rightarrow J(f)$, there exists a unique equilibrium state for $\psi$ and a unique $\exp (\mathrm{P}(\psi)-\psi)$ conformal measure in the sense of $[5]$, where $\mathrm{P}(\psi)$ denotes the pressure of $\left(\left.f\right|_{J(f)}, \psi\right)$.

(2) Moreover, $\mathrm{P}(\psi)=\mathrm{P}^{p}(\psi)$ and for each point $x \in J(f)$, we have that $\frac{1}{n} \log \mathcal{L}_{\psi}^{n} \mathbb{1}(x) \rightarrow \mathrm{P}(\psi)=\mathrm{P}^{p}(\psi)$ as $n \rightarrow \infty$, where $\mathcal{L}_{\psi}$ denotes the Perron-Frobenius operator associated with the potential $\psi$ (see (4.1)) and $\mathbb{1} \equiv 1$. 
Remark 1.2. If $\psi: J(f) \rightarrow \mathbb{R}$ is a Hölder continuous function such that $\sup (\psi)-\inf (\psi)<\log \left(\sum_{j=1}^{s} e_{j}\right)-\log s$, then $\mathrm{P}^{p}(\psi)>\sup (\psi)+\log s$.

Remark 1.3. Under a very mild condition, the topological entropy $h(f)$ of $f: \Sigma_{s} \times \overline{\mathbb{C}} \rightarrow \Sigma_{s} \times \overline{\mathbb{C}}$ is equal to $\log \left(\sum_{j=1}^{s} e_{j}\right)$ and there exists a unique maximal entropy measure for $f: \Sigma_{s} \times \overline{\mathbb{C}} \rightarrow \Sigma_{s} \times \overline{\mathbb{C}}$ (See [13]).

The proof of the main result is given in the following several sections. In this proof we utilize some arguments from [5] to show the existence of a conformal measure. Then, using the normality of a family of inverse branches of elements of the semigroup (see section 2), we analyze the Perron-Frobenius operator in detail. Developing the techniques worked out in [6] and [13], we show the existence of an equilibrium state and the uniqueness of a conformal measure as well as an equilibrium state.

\section{Distortion Theorems}

Let us recall the following well-known version of Koebe's Distortion Theorem concerning spherical derivatives.

Theorem 2.1. For every $u \in(0, \operatorname{diam}(\overline{\mathscr{C}}) / 2)$ there exists a function $k_{u}:[0,1) \rightarrow(0,+\infty)$, continuous at 0 , with $k_{u}(0)=1$ and the following property. If $\xi \in \overline{\mathbb{C}}, R>0$, and $H: B(\xi, R) \rightarrow \overline{\mathbb{C}}$ is a meromorphic univalent function such that $\overline{\mathbb{C}} \backslash H(B(\xi, R))$ contains a ball of radius $u$, then for every $t \in[0,1)$ and all $z, w \in B(\xi, t R)$,

$$
k_{u}^{-1}(t) \leq \frac{\left|H^{\prime}(w)\right|}{\left|H^{\prime}(z)\right|} \leq k_{u}(t) .
$$

As an immediate consequence of this theorem, combined with Lemma 4.5 in [13], we get the following.

Lemma 2.2. Let $G=\left\langle f_{1}, \ldots, f_{m}\right\rangle$ be a finitely generated rational semigroup satisfying the condition (E1). Then, there exists a number $R_{0}>0$ and a function $k_{G}:[0,1) \times\left(0, R_{0}\right] \rightarrow[1, \infty)$ such that for each $x \in$ $J(G), 0<R \leq R_{0}$ and $0 \leq t<1$, the family $\mathcal{F}_{x, R}:=\{\varphi: B(x, R) \rightarrow$ 
$\overline{\mathbb{C}} \mid \varphi$ is a well-defined inverse branch of $h, h \in G\}$ satisfies that for each $H \in \mathcal{F}_{x, R}, w, z \in B(x, t R)$, we have

$$
k_{G}(t, R)^{-1} \leq \frac{\left|H^{\prime}(w)\right|}{\left|H^{\prime}(z)\right|} \leq k_{G}(t, R) .
$$

Proof. By Lemma 4.5 in [13], we have that there exists a number $R_{0}>0$ such that for each $x \in J(G)$ and each $0<R \leq R_{0}$, the family $\mathcal{F}_{x, R}$ is normal in $B(x, R)$. Now, suppose the statement of our lemma is false. Then, there exist a $0<t<1$, a sequence $\left(x_{n}\right)$ in $J(G)$, a sequence $\left(w_{n}\right)$ in $\overline{\mathbb{C}}$, a sequence $\left(z_{n}\right)$ in $\overline{\mathbb{C}}$, a number $0<R \leq R_{0}$, and a sequence $\left(\varphi_{n}\right)$ of meromorphic functions, such that for each $n \in \mathbb{N}$, we have $w_{n}, z_{n} \in B\left(x_{n}, t R\right), \varphi_{n} \in \mathcal{F}_{x_{n}, R}$, and $\frac{\left|\varphi_{n}^{\prime}\left(w_{n}\right)\right|}{\left|\varphi_{n}^{\prime}\left(z_{n}\right)\right|} \geq n$. We may assume $x_{n} \rightarrow x_{\infty} \in J(G), w_{n} \rightarrow w_{\infty} \in \overline{\mathbb{C}}$, and $z_{n} \rightarrow z_{\infty} \in \overline{\mathbb{C}}$. Let $u$ be a number with $t<u<1$. Then there exists an $n_{0} \in \mathbb{N}$ such that each $\varphi_{n}\left(n \geq n_{0}\right)$ is defined on $B\left(x_{\infty}, u R\right)$ and the family $\left\{\varphi_{n}\right\}_{n \geq n_{0}}$ is normal in $B\left(x_{\infty}, u R\right)$. Then we may assume that $\varphi_{n}$ tends to a meromorphic function $\varphi_{\infty}: B\left(x_{\infty}, u R\right) \rightarrow \overline{\mathbb{C}}$ as $n \rightarrow \infty$, uniformly on $B\left(x_{\infty}, u R\right)$. Since each $\varphi_{n}$ is injective, we have that $\varphi_{\infty}: B\left(x_{\infty}, u R\right) \rightarrow \overline{\mathbb{C}}$ is either injective or constant. Hence, $\varphi_{\infty}\left(B\left(x_{\infty}, u R\right)\right) \neq \overline{\mathbb{C}}$. Let $v$ be a number with $t<v<u$. Then, there exist a number $0<s<1$ and a point $a \in \overline{\mathbb{C}}$ such that for each large $n \geq n_{0}$,

$$
B(a, s) \subset \overline{\mathbb{C}} \backslash \varphi_{n}\left(B\left(x_{\infty}, v R\right)\right) .
$$

Then, by Theorem 2.1, it causes a contradiction. We are done.

Notation. Throughout the rest of the paper, we set $K_{G}(R):=k_{G}\left(\frac{1}{2}, R\right)$.

\section{INVERSE BRANCHES}

We set $\Sigma_{s}^{*}:=\cup_{j=1}^{\infty}\{1, \ldots, s\}^{j}$ (disjoint union). For each $\omega \in \Sigma_{s}^{*}$, we set $|\omega|=j$ if $\omega \in\{1, \ldots, s\}^{j}$. By $\hat{\Sigma}_{s}^{+}$we denote the space dual to $\Sigma_{s}$, that is $\hat{\Sigma}_{s}^{+}$consists of infinite sequences $\omega=\ldots \omega_{3} \omega_{2} \omega_{1}$ of elements from the set $\{1,2, \ldots, s\}$. For each $\omega \in \hat{\Sigma}_{s}^{+}$, by $\left.\omega\right|_{n}$ we denote the finite word $\left(\omega_{n}, \omega_{n-1}, \ldots, \omega_{2}, \omega_{1}\right)$; more generally, for $b \geq a$ we put $\left.\omega\right|_{b} ^{a}=$ $\left(\omega_{b}, \omega_{b-1}, \ldots, \omega_{a}\right)$. For each finite word $\omega=\left(\omega_{n}, \ldots, \omega_{1}\right)$, we set $f_{\omega}:=$ $f_{\omega_{1}} \circ \cdots \circ f_{\omega_{n}}$. The technical tool that allows us to develop the further machinery is the following.

Lemma 3.1. Let $G=\left\langle f_{1}, \ldots, f_{s}\right\rangle$ be a rational semigroup satisfying the condition (E1). Let $R_{0}$ be the number in Lemma 2.2. Fix an integer 
$q \geq 1$ and a real number $\lambda \in(0,1)$. Then for every finite set $E \subset J(G)$, every $\omega \in \hat{\Sigma}_{s}^{+}$, every

$R \in$ $\left(0, \min \left\{1, \frac{1}{2} R_{0}, \kappa^{-1 / 2}, \frac{1}{2} \operatorname{dist}\left(E, C V\left(f_{\left.\omega\right|_{q}}\right)\right), 4^{-1} \min \{d(z, \xi): z, \xi \in E, z \neq \xi\}\right\}\right)$, every integer $n \geq 0$, and every $z \in E$, there exists a subset $I_{n}(z, \omega)$ of the set of all inverse meromorphic branches of $f_{\left.\omega\right|_{q n}}$ defined on $B(z, 2 R)$ and satisfying the following properties with $I_{n}=\bigcup_{z \in E} I_{n}(z, \omega)$.

$\left(a_{n}\right)$ If $z \in E$ and $\phi \in I_{n+1}(z, \omega)$, then $f_{\left.\omega\right|_{q(n+1)} ^{q n+1}} \circ \phi \in I_{n}(z, \omega)$.

$\left(b_{n}\right)$ If $\phi \in I_{n}$, then $\operatorname{diam}(\phi(B(z, R))) \leq K_{G}(2 R)^{2} \kappa^{-1 / 2} \lambda^{n / 2} C_{s a}^{1 / 2}$.

$\left(c_{n}\right) \phi(B(z, 2 R)) \cap C V\left(f_{\left.\omega\right|_{q(n+1)} ^{q n+1}}\right)=\emptyset$ for all $z \in E$ and all $\phi \in I_{n}$.

$\left(d_{n}\right) \#\left(J_{n} \backslash I_{n}\right) \leq d q+\lambda^{-n}$ for all $n \geq 1$, where $J_{n}$ is the family of all compositions of all maps $\phi \in I_{n-1}(z, \omega), z \in E$, with all meromorphic inverse branches of $f_{\left.\omega\right|_{q n} ^{q(n-1)+1}}$.

(e) $I_{0}=\left\{\left.\operatorname{Id}\right|_{B(z, 2 R)} \mid z \in E\right\}$.

Proof. We shall construct recursively the sets $I_{n}(z, \omega), n \geq 0$, such that the conditions $\left(a_{n}\right),\left(b_{n}^{\prime}\right),\left(c_{n}\right)$ and $\left(d_{n}\right)$ (here $\left.n \geq 1\right)$, where $\left(b_{n}^{\prime}\right)$ requires that

$$
\left(b_{n}^{\prime}\right) \text { If } \phi \in I_{n} \text {, then } A(\phi(B(z, R))) \leq \lambda^{n} .
$$

The base of induction, the family $I_{0}$ consists of all the identity maps defined on the balls $B(z, 2 R), z \in E$. The condition $\left(b_{0}^{\prime}\right)$ is satisfied since $A(\overline{\mathscr{C}})=1$ and $\left(c_{0}\right)$ is satisfied because of the choice of the radius $R$. Now assume that for some $n \geq 0$ the subsets $I_{n}(z, \omega), z \in E$, have been constructed so that the conditions $\left(b_{n}^{\prime}\right)$ and $\left(c_{n}\right)$ are satisfied. The inductive step is to construct the subsets $I_{n+1}(z, \omega), z \in E$, so that the conditions $\left(a_{n+1}\right),\left(b_{n+1}^{\prime}\right),\left(c_{n+1}\right)$ and $\left(d_{n+1}\right)$ are satisfied. This will complete our recursive construction. In view of $\left(c_{n}\right)$ all the meromorphic inverse branches of $f_{\left.\omega\right|_{q(n+1)} ^{q n+1}}$ are well defined on all the sets $\phi(B(z, 2 R)$ ), $z \in E, \phi \in I_{n}(z, \omega)$. Their compositions with corresponding elements $\phi \in I_{n}(z, \omega)$ are said to form the subset $J_{n+1}(z, \omega)$. Note that $J_{n+1}=$ $\bigcup_{z \in E} J_{n+1}(z, \omega)$. The subset $I_{n+1}(z, \omega), z \in E$, is defined to consist of all the elements $\psi \in J_{n+1}(z, \omega)$ for which the following two conditions are satisfied.

(i) $A(\psi(B(z, R))) \leq \lambda^{n+1}$.

(ii) $\psi(B(z, 2 R)) \cap C V\left(f_{\left.\omega\right|_{q(n+2)} ^{q(n+1)+1}}\right)=\emptyset$.

Thus, conditions $\left(b_{n+1}^{\prime}\right)$ and $\left(c_{n+1}\right)$ are satisfied immediately. Condition $\left(a_{n}\right)$ is satisfied since it holds for all $\psi \in J_{n+1}(z, \omega), z \in E$, and $I_{n+1}(z, \omega)$ is a subset of $J_{n+1}(z, \omega)$. We are left to show that $\left(d_{n+1}\right)$ 
holds. Now, if $\psi \in J_{n+1}$, say $\psi \in J_{n+1}(z, \omega), z \in E$, but (i) is not satisfied, then $A(\psi(B(z, R)))>\lambda^{n+1}$. Since all the sets $\psi(B(z, R))$, $\psi \in J_{n+1}(z, \omega), z \in E$, are mutually disjoint and since $A(\overline{\mathscr{C}})=1$, we conclude that the number of elements of $J_{n+1}$ for which condition (i) fails is bounded above by $1 / \lambda^{n+1}=\lambda^{-(n+1)}$. Since the number of critical points of each generator of the semigroup $G$ is bounded above by $d$, the cardinality of the set of critical values of $f_{\left.\omega\right|_{q(n+2} ^{q(n+1)+1}}$ is bounded above by $d q$. Since all the sets $\psi(B(z, 2 R)), \psi \in J_{n+1}(z, \omega), z \in E$, are mutually disjoint, we thus conclude that the number of elements $\psi \in J_{n+1}$ for which condition (ii) fails, is bounded above by $d q$. In conclusion \# $\left(J_{n+1} \backslash I_{n+1}\right) \leq d q+\lambda^{-(n+1)}$, meaning that $\left(d_{n+1}\right)$ is satisfied. The recursive construction is complete. Since $G$ satisfies (E1), it follows from Lemma 2.2 that for all $n \geq 0$, all $z \in E$, and all $\phi \in I_{n}(z, \omega)$, we have

$$
B\left(\phi(z), K_{G}(2 R)^{-1}\left|\phi^{\prime}(z)\right| R\right) \subset \phi(B(z, R)) \subset B\left(\phi(z), K_{G}(2 R)\left|\phi^{\prime}(z)\right| R\right) .
$$

Hence, making use of $\left(b_{n}^{\prime}\right)$, we get that

$$
\begin{aligned}
\operatorname{diam}^{2}(\phi(B(z, R))) & \leq\left(2 K_{G}(2 R)\left|\phi^{\prime}(z)\right| R\right)^{2} \\
& =K_{G}(2 R)^{4} \kappa^{-1}\left(2 \kappa^{1 / 2} K_{G}(2 R)^{-1}\left|\phi^{\prime}(z)\right| R\right)^{2} \\
& =K_{G}(2 R)^{4} \kappa^{-1} C_{s a} A\left(B\left(\phi(z), K_{G}(2 R)^{-1}\left|\phi^{\prime}(z)\right| R\right)\right) \\
& \leq K_{G}(2 R)^{4} \kappa^{-1} C_{s a} A(\phi(B(z, R))) \\
& \leq K_{G}(2 R)^{4} \kappa^{-1} C_{s a} \lambda^{n} .
\end{aligned}
$$

Thus, $\operatorname{diam}(\phi(B(z, R))) \leq K_{G}(2 R)^{2} \kappa^{-1 / 2} C_{s a}^{1 / 2} \lambda^{n / 2}$. We are done.

In order to simplify the notation, put

$$
\begin{aligned}
& R\left(E,\left.\omega\right|_{q}\right):= \\
& \min \left\{1, \frac{1}{2} R_{0}, \kappa^{-1 / 2}, \frac{1}{2} \operatorname{dist}\left(E, C V\left(f_{\left.\omega\right|_{q}}\right)\right), 4^{-1} \min \{d(z, \xi): z, \xi \in E, z \neq \xi\}\right\} .
\end{aligned}
$$

Corollary 3.2. Suppose that $G=\left\langle f_{1}, \ldots, f_{s}\right\rangle$ is an E-semigroup of rational maps. Fix an integer $q \geq 1$ and a real number $\lambda \in(0,1)$. Then for every $z \in J(G)$, there exists a number $R=R_{q}(z)>0$, a number $R_{q}^{\prime}(z)>0$, and a number $D \geq 1$, where $D$ does not depend on $q, \lambda, z$, such that for every $\omega \in \hat{\Sigma}_{s}^{+}$and every integer $n \geq 1$, there exists $W_{n}(z, \omega), Z_{n}(z, \omega)$, a subset of the set of all connected components of $f_{\left.\omega\right|_{q n}}^{-1}(B(z, R))$, with the following properties. 
$\left(A_{n}\right)$ If $V \in W_{n+1}(z, \omega)$, then $f_{\left.\omega\right|_{q(n+1)} ^{q n+1}}(V) \in W_{n}(z, \omega)$.

$\left(B_{n}\right)$ If $V \in W_{n}(z, \omega)$, then $\operatorname{diam}(V) \leq K_{G}\left(R_{q}^{\prime}(z)\right)^{4} \kappa^{-1 / 2} \lambda^{n / 2} C_{s a}^{1 / 2}$.

$\left(C_{n}\right) Z_{n}(z, \omega)$ is the family of all connected components of the sets $f_{\left.\omega\right|_{q n} ^{q(n-1)+1}}^{-1}(V), V \in W_{n-1}(z, \omega)$, and $\#\left(Z_{n}(z, \omega) \backslash W_{n}(z, \omega)\right) \leq$ $d q+\lambda^{-n}$, and

$\left(D_{n}\right)$ For every $V \in Z_{n}(z, \omega)$, the map $\left.f_{\left.\omega\right|_{q n}}\right|_{V}: V \rightarrow \overline{\mathbb{C}}$ is at most D-to-1.

$\left(E_{1}\right) W_{1}(z, \omega)=Z_{1}(z, \omega)=\left\{\right.$ connected components $V$ of $\left.f_{\left.\omega\right|_{q}}^{-1}(B(z, R))\right\}$.

Note also that in fact $W_{n}(z, \omega)$ depends only on $z$ and $\left.\omega\right|_{q n}$, so we can and will in the forthcoming sections write $W_{n}\left(z,\left.\omega\right|_{q n}\right)$

Proof. Let $z \in J(G)$ be a point. Since $J(G)=\pi_{2}(J(f))$, there exists a point $\omega \in \Sigma_{s}$ such that $(\omega, z) \in J(f)$. Then, from the assumption E2, E3, there exists $p \geq 1$ independent of $q$ (but depending on $(\omega, z)$ ) such that

Then, we obtain

$$
\left(\bigcup_{r \in \mathbb{N}}\left(f^{p q+r}\right)^{-1}(\omega, z)\right) \cap \operatorname{Crit}(f)=\emptyset
$$

$$
\bigcup_{\rho \in \Sigma_{s}^{*}|\tau|=p q} \bigcup_{\rho}^{-1}\left(f_{\tau}^{-1}(z)\right) \cap \operatorname{Crit}\left(f_{\rho}\right)=\emptyset .
$$

In particular

$$
\left(\bigcup_{|\tau|=p q} f_{\tau}^{-1}(z)\right) \cap C V\left(f_{\rho}\right)=\emptyset
$$

for all $\rho \in \Sigma_{s}^{*}$. Set $E=\bigcup_{|\tau|=p q} f_{\tau}^{-1}(z)$. It follows from (3.1) that

$$
\hat{R}_{q}(z)=\frac{1}{2} \lambda^{p / 2} \cdot \min \left\{R(E, \rho): \rho \in\{1,2, \ldots, s\}^{q}\right\}>0 .
$$

Now, there is $R_{q}(z)>0$ so small that the following two conditions are satisfied.

(a) For each $\tau \in\{1,2, \ldots, s\}^{p q}$ each connected component of $f_{\tau}^{-1}\left(B\left(z, R_{q}(z)\right)\right)$ is contained in exactly one ball $B\left(\xi, \hat{R}_{q}(z)\right)$, where $\xi \in E$.

(b) For each $\gamma \in \Sigma_{s}^{*}$ with $|\gamma| \leq p q$, each connected component of $f_{\gamma}^{-1}\left(B\left(z, R_{q}(z)\right)\right)$ has the diameter bounded above by $\kappa^{-1 / 2} \lambda^{|\gamma| / 2}$.

Now, for every $1 \leq k \leq p$ and every $\omega \in \hat{\Sigma}_{s}^{+}$, define $W_{k}(z, \omega)$ and $Z_{k}(z, \omega)$ to be the family of all connected components of $f_{\left.\omega\right|_{q k}}^{-1}\left(B\left(z, R_{q}(z)\right)\right)$. The conditions $\left(A_{k}\right),\left(B_{k}\right)$, and $\left(C_{k}\right)$ are obviously satisfied for all $1 \leq$ $k \leq p$. Now, for every $\omega \in \hat{\Sigma}_{s}^{+}$, every $n \geq p+1$ and every $\xi \in$ 
$f_{\omega \mid p q}^{-1}(z) \subset E$, consider the inverse branch $\phi: B\left(\xi, 2 \lambda^{-p / 2} \hat{R}_{q}(z)\right) \rightarrow \overline{\mathbb{C}} \in$ $I_{n-p}\left(\xi,\left.\omega\right|_{\infty} ^{p q}\right)$. It follows from Lemma $3.1\left(b_{n-p}\right)$ and Lemma 2.2 that

$$
\begin{aligned}
2 K_{G}\left(2 \lambda^{-\frac{p}{2}} \hat{R}_{q}(z)\right)^{-1} \lambda^{-p / 2}\left|\phi^{\prime}(\xi)\right| \hat{R}_{q}(z) & \leq \operatorname{diam}\left(\phi\left(B\left(\xi, \lambda^{-p / 2} \hat{R}_{q}(z)\right)\right)\right) \\
& \leq K_{G}\left(2 \lambda^{-\frac{p}{2}} \hat{R}_{q}(z)\right)^{2} \kappa^{-1 / 2} \lambda^{\frac{n-p}{2}} C_{s a}^{1 / 2} .
\end{aligned}
$$

Thus $\left|\phi^{\prime}(\xi)\right| \leq \frac{1}{2} K_{G}\left(2 \lambda^{-\frac{p}{2}} \hat{R}_{q}(z)\right)^{3} \kappa^{-1 / 2} \lambda^{n / 2} \hat{R}_{q}(z)^{-1} C_{s a}^{1 / 2}$, and therefore, using Lemma 2.2 again and the definition of $\hat{R}_{q}(z)$, we get that

$$
\operatorname{diam}\left(\phi\left(B\left(\xi, \hat{R}_{q}(z)\right)\right)\right) \leq K_{G}\left(2 \lambda^{-\frac{p}{2}} \hat{R}_{q}(z)\right)^{4} \kappa^{-1 / 2} \lambda^{n / 2} C_{s a}^{1 / 2} .
$$

So, looking also at Lemma $3.1\left(d_{n}\right)$ and $\left(c_{n}\right)$, we complete the proof of items $\left(A_{n}\right),\left(B_{n}\right)$ and $\left(C_{n}\right)$ by defining for every $n \geq p+1$ the family $W_{n}(z, \omega)$ to consist of all the sets of the form $\phi\left(V_{\xi}\right)$, where $\xi \in f_{\left.\omega\right|_{p q}}^{-1}(z), V_{\xi}$ is a connected component of $f_{\left.\omega\right|_{p q}}^{-1}\left(B\left(z, R_{q}(z)\right)\right)$ contained in $B\left(\xi, \hat{R}_{q}(z)\right)$, and $\phi \in I_{n-p}\left(\xi, \omega_{\infty}^{p q}\right)$. Decreasing $R_{q}(z)$ appropriately, the items $\left(D_{n}\right)$ follow now from this construction and Lemma 3.1.

\section{Perron-Frobenius Operators and Gibbs States}

From now on throughout the entire paper assume that $\psi: J(f) \rightarrow \mathbb{R}$ is a Hölder continuous function. Given $n \geq 1, \omega \in\{1, \ldots, s\}^{n}$, and a continuous function $g: J(f) \rightarrow \mathbb{R}$, define

$$
\mathcal{L}_{\psi, \omega}^{(n)} g(\tau, z)=\sum_{x \in f_{\omega}^{-1}(z)} \exp \left(S_{n} \psi(\omega \tau, x)\right) g(\omega \tau, x),
$$

where here and in the sequel the summation is taken with multiplicities of all critical points of $f_{\omega}$, and $S_{n} \psi:=\sum_{j=0}^{n-1} \psi \circ f^{j}$. Since $\sup \left\{\#\left(f_{\omega}^{-1}(z)\right)\right.$ : $z \in \mathbb{C}\}<\infty, \mathcal{L}_{\psi, \omega}^{(n)}$ is a bounded linear operator acting on the Banach space $C(J(f))$ of continuous functions on $J(f)$ endowed with the supremum norm. Set

$$
\mathcal{L}_{\psi}^{(n)}:=\sum_{|\omega|=n} \mathcal{L}_{\psi, \omega}^{(n)}, \mathcal{L}_{\psi}:=\mathcal{L}_{\psi}^{(1)}
$$

Then $\mathcal{L}_{\psi}^{(n)}$ and $\mathcal{L}_{\psi}$ also act continuously on the Banach space $C(J(f))$. We call $\mathcal{L}_{\psi}: C(J(f)) \rightarrow C(J(f))$ the Perron-Frobenius operator associated with the potential $\psi$. Note that

$$
\mathcal{L}_{\psi}^{(n)} g(x)=\sum_{y \in f^{-n}(x)} \exp \left(S_{n} \psi(y)\right) g(y)=\mathcal{L}_{\psi}^{n} g(x) .
$$


Define the pointwise pressure $\mathrm{P}^{p}(\psi)$ of the function $\psi$ by the following formula.

$$
\begin{aligned}
& \mathrm{P}_{x}(\psi):=\limsup _{n \rightarrow \infty} \frac{1}{n} \log \mathcal{L}_{\psi}^{n} \mathbb{1}(x), x \in J(f), \quad \text { and } \\
& \mathrm{P}^{p}(\psi):=\sup \left\{\mathrm{P}_{x}(\psi): x \in J(f)\right\},
\end{aligned}
$$

where $\mathbb{1}(x):=1$. Throughout the entire paper we work with the assumption that

$$
\mathrm{P}^{p}(\psi)>\sup (\psi)+\log s
$$

In particular, we can fix a point $b \in J(f)$ such that

$$
\eta:=\exp \left(\sup (\psi)+\log s-\mathrm{P}_{b}(\psi)\right)<1 .
$$

Fix $\lambda \in(0,1)$. There then exists $q=q(\lambda) \geq 1$ such that

$$
\gamma_{q}^{-1}:=d q+\lambda^{-1} \leq(1+2 D)^{-1} \eta^{-q} .
$$

Let $\mathcal{L}_{\psi}^{*}: C(J(f))^{*} \rightarrow C(J(f))^{*}$ be the operator conjugate to $\mathcal{L}_{\psi}$, i.e., $\mathcal{L}_{\psi}^{*} \nu(g)=\nu\left(\mathcal{L}_{\psi} g\right)$. Using (4.4) and the fact that the map $f: J(f) \rightarrow$ $J(f)$ is open, as an immediate consequence of Theorem 3.9 and Proposition 2.2 in [5], we get the following.

Lemma 4.1. If $G=\left\langle f_{1}, \ldots, f_{s}\right\rangle$ is an E-semigroup of rational maps, $\psi: J(f) \rightarrow \mathbb{R}$ is a Hölder continuous potential satisfying (4.3) and $b \in J(f)$ is selected so that (4.4) holds, then there exists a Borel atomless probability measure $m_{\psi}$ on $J(f)$ such that $\mathcal{L}_{\psi}^{*} m_{\psi}=e^{\mathrm{P}_{b}(\psi)} m_{\psi}$.

The measure $m_{\psi}$ is called $\exp \left(\mathrm{P}_{b}(\psi)-\psi\right)$-conformal for $f$. Obviously $\left(\mathcal{L}_{\psi}^{*}\right)^{n} m_{\psi}=e^{\mathrm{P}_{b}(\psi) n} m_{\psi}$ for all $n \geq 0$, and this equivalently means that

$$
m_{\psi}\left(f^{n}(A)\right)=\int_{A} \exp \left(\mathrm{P}_{b}(\psi) n-S_{n} \psi\right) d m_{\psi}
$$

for every Borel set $A \subset J(f)$ for which the restriction $\left.f^{n}\right|_{A}$ is injective.

Remark 4.2. Note that all forthcoming considerations depend only on the above relation and not on the particular way the measure $m_{\psi}$ was constructed.

From now on throughout the paper put

$$
\bar{\psi}=\psi-\mathrm{P}_{b}(\psi)
$$


Now set

$$
L_{\omega}^{(n)}=\mathcal{L}_{\bar{\psi}, \omega}^{(n)}, \mathcal{L}^{n}=\mathcal{L}_{\bar{\psi}}^{n}, \quad \text { and } \quad \mathcal{L}=\mathcal{L}_{\bar{\psi}}
$$

Now for every $z \in J(G)$, every $n \geq 1$, every $\omega \in\{1, \ldots, s\}^{q n}$, and every $g \in C(J(f))$, define the function $G_{z, \omega}^{n} g: \pi_{2}^{-1}\left(B\left(z, R_{q}(z)\right)\right) \rightarrow \mathbb{R}$ by setting

$$
G_{z, \omega}^{n} g(\tau, \xi)=\sum_{V \in W_{n}(z, \omega)} \sum_{x \in f_{\omega}^{-1}(\xi) \cap V} \exp \left(S_{q n} \bar{\psi}(\omega \tau, x)\right) g(\omega \tau, x),
$$

where $R_{q}(z)$ and $W_{n}(z, w)$ come from Corollary 3.2. Since for every $V \in W_{n}(z, \omega)$ the map $f_{\omega}: V \rightarrow B\left(z, R_{q}(z)\right)$ is a branched covering, for every $\xi \in B\left(z, R_{q}(z)\right)$ there is a bijection $\hat{\xi}: f_{\omega}^{-1}(z) \cap V \rightarrow f_{\omega}^{-1}(\xi) \cap V$, where all critical points of $f_{\omega}$ in $f_{\omega}^{-1}(z)$ and $f_{\omega}^{-1}(\xi)$ are counted with multiplicities. So, (4.6) can be rewritten in the following form.

$$
G_{z, \omega}^{n} g(\tau, \xi)=\sum_{V \in W_{n}(z, \omega)} \sum_{x \in f_{\omega}^{-1}(z) \cap V} \exp \left(S_{q n} \bar{\psi}(\omega \tau, \hat{\xi}(x))\right) g(\omega \tau, \hat{\xi}(x)) .
$$

Since the function $\psi: J(f) \rightarrow \mathbb{R}$ is Hölder continuous, it follows from Corollary $3.2\left(B_{n}\right)$ that there exists a constant $H>0$ such that (with $\left.x \in f_{\omega}^{-1}(z) \cap V\right)$,

$$
\left|S_{q n} \bar{\psi}(\omega \tau, \hat{\xi}(x))-S_{q n} \bar{\psi}(\omega \theta, x)\right| \leq H
$$

for all $\tau, \theta \in \Sigma_{s}$, or equivalently,

$$
e^{-H} \exp \left(S_{q n} \bar{\psi}(\omega \theta, x)\right) \leq \exp \left(S_{q n} \bar{\psi}(\omega \tau, \hat{\xi}(x))\right) \leq e^{H} \exp \left(S_{q n} \bar{\psi}(\omega \theta, x)\right) \text {. }
$$

In consequence

$$
e^{-H} G_{z, \omega}^{n} \mathbb{1}(\theta, z) \leq G_{z, \omega}^{n} \mathbb{1}(\tau, \xi) \leq e^{H} G_{z, \omega}^{n} \mathbb{1}(\theta, z) .
$$

Set

$$
G_{z}^{n}=\sum_{|\omega|=q n} G_{z, \omega}^{n}
$$

It then follows from (4.8) that

$$
e^{-H} G_{z}^{n} \mathbb{1}(\theta, z) \leq \sum_{|\omega|=q n} G_{z, \omega}^{n} \mathbb{1}\left(\tau_{\omega}, \xi_{\omega}\right) \leq e^{H} G_{z}^{n} \mathbb{1}(\theta, z)
$$

for all $n \geq 0$, all $(\theta, z) \in J(f)$ and all $\left(\tau_{\omega}, \xi_{\omega}\right) \in \pi_{2}^{-1}\left(B\left(z, R_{q}(z)\right)\right)$. Since $J(G)$ is a compact set, there exist finitely many points, say $z_{1}, z_{2}, \ldots, z_{u} \in$ $J(G)$ such that $\bigcup_{j=1}^{u} B\left(z_{j}, R_{q}\left(z_{j}\right)\right) \supset J(G)$. Put $M_{j, \omega}^{n} g=\left.L_{\omega}^{(q n)}\right|_{\pi_{2}^{-1}\left(B\left(z_{j}, R_{q}\left(z_{j}\right)\right)\right)}, M_{j}^{n} g=\sum_{|\omega|=q n} M_{j, \omega}^{n} g, G_{j, \omega}^{n}=G_{z_{j}, \omega}^{n}, G_{j}^{n}=G_{z_{j}}^{n}$, 
and

$$
\begin{aligned}
\left\|G_{j}^{n} g \mid\right\|_{\infty} & =\sum_{|\omega|=q n}\left\|G_{j, \omega}^{n} g\right\|_{\infty},\left\||| M_{j}^{n} g\right\|_{\infty}=\sum_{|\omega|=q n}\left\|M_{j, \omega}^{n} g\right\|_{\infty}, \\
\left\|\mid \mathcal{L}^{n} g\right\| \|_{\infty} & =\sum_{|\omega|=n}\left\|L_{\omega}^{(n)} g\right\|_{\infty} .
\end{aligned}
$$

We shall prove the following.

\section{Lemma 4.3. It holds}

$$
0<Q_{\psi}:=\max _{1 \leq j \leq u} \sup _{n \geq 0}\left\{||\left|G_{j}^{n} \mathbb{1}\right| \|_{\infty}\right\}<\infty .
$$

Proof. Since the map $f: J(f) \rightarrow J(f)$ is topologically exact and since $\left\{B\left(z_{j}, R_{q}\left(z_{j}\right)\right)\right\}_{j=1}^{u}$ is an open cover of $J(f)$, there exists $k \geq 1$ such that for all $j=1,2, \ldots, u$,

$$
f^{k q}\left(\pi_{2}^{-1}\left(B\left(z_{j}, R_{q}\left(z_{j}\right)\right)\right)\right) \supset J(f) .
$$

By Lemma $4.1, \mathcal{L}^{* n} m_{\psi}=m_{\psi}$ for all $n \geq 0$, and consequently $\int \mathcal{L}^{n} \mathbb{1} d m_{\psi}=$ $\int \mathbb{1} d m_{\psi}=1$. Fix $n \geq 0$. There then exists $w_{0} \in J(f)$ such that

$$
\mathcal{L}^{q(k+n)} \mathbb{1}\left(w_{0}\right) \leq 1 .
$$

Now fix an arbitrary $1 \leq j \leq u$, an arbitrary $\omega \in\{1, \ldots, s\}^{q n}$, and an arbitrary $x_{\omega} \in \pi_{2}^{-1}\left(B\left(z_{j}, R_{q}\left(z_{j}\right)\right)\right)$. By (4.10) there exists $y_{j} \in$ $\pi_{2}^{-1}\left(B\left(z_{j}, R_{q}\left(z_{j}\right)\right)\right)$ such that $f^{k q}\left(y_{j}\right)=w_{0}$. Applying (4.9) with $z=z_{j}$, we get that

$$
\sum_{|\omega|=q n} G_{j, \omega}^{n} \mathbb{1}\left(x_{\omega}\right) \leq e^{2 H} G_{j}^{n} \mathbb{1}\left(y_{j}\right) .
$$

Also, by (4.11), we obtain

$$
\begin{aligned}
G_{j}^{n} \mathbb{1}\left(y_{j}\right) & \leq \mathcal{L}^{q n} \mathbb{1}\left(y_{j}\right) \leq\left\|\exp \left(S_{k q}(-\bar{\psi})\right)\right\|_{\infty} \mathcal{L}^{q(k+n)} \mathbb{1}\left(w_{0}\right) \\
& \leq\left\|\exp \left(S_{k q}(-\bar{\psi})\right)\right\|_{\infty} .
\end{aligned}
$$

Thus, $\sum_{|\omega|=q n} G_{j, \omega}^{n} \mathbb{1}\left(x_{\omega}\right) \leq e^{2 H}|| \exp \left(S_{k q}(-\bar{\psi})\right) \|_{\infty}$, and we are done by taking supremum over all $x_{\omega} \in \pi_{2}^{-1}\left(B\left(z_{j}, R_{q}\left(z_{j}\right)\right)\right)$.

Now we are in position to prove the required upper bound on the iterates of the Perron-Frobenius operator $\mathcal{L}$. 
Lemma 4.4. For every $n \geq 1$, we have that

$$
\begin{aligned}
\left\|\left|\mathcal{L}^{q n} \mathbb{1}\right|\right\|_{\infty} & \leq Q_{\psi}+D \sum_{k=1}^{n}\left(\eta^{q} \gamma_{q}^{-1}\right)^{k} \mid\left\|\mathcal{L}^{q(n-k)} \mathbb{1}\right\| \|_{\infty} \\
& \leq Q_{\psi}+D \sum_{k=1}^{n}(1+2 D)^{-k}\left\|\mid \mathcal{L}^{q(n-k)} \mathbb{1}\right\|_{\infty}
\end{aligned}
$$

Proof. Fix $n \geq 1, \omega=\left(\omega_{q n}, \omega_{q n-1}, \ldots, \omega_{1}\right) \in\{1, \ldots, s\}^{q n}$, and $(\tau, x) \in$ $J(f)$. There then exists $1 \leq j \leq u$ such that $(\tau, x) \in \pi_{2}^{-1}\left(B\left(z_{j}, R_{q}\left(z_{j}\right)\right)\right)$. For each $a, b \in \mathbb{N}$ with $a \leq b \leq q n$, we set $\left.\omega\right|_{b} ^{a}:=\left(\omega_{b}, \ldots, \omega_{a}\right)$. Moreover, for each $l \in \mathbb{N}$ with $l \leq q n$, we set $\left.\omega\right|_{l}=\left(\omega_{l}, \ldots, \omega_{1}\right)$.

One can now represent $L_{\omega}^{(q n)} \mathbb{1}(\tau, x)$ in the following way.

$$
\begin{aligned}
& M_{j, \omega}^{n} \mathbb{1}(\tau, x) \\
& =\sum_{k=1}^{n} \sum_{V \in Z_{k}\left(z_{j}, \omega\right) \backslash W_{k}\left(z_{j}, \omega\right)} \sum_{y \in V \cap f_{\left.\omega\right|_{q k}}^{-1}(x)} \exp \left(S_{q k} \bar{\psi}\left(\left.\omega\right|_{q k} \tau, y\right)\right) L_{\left.\omega\right|_{q n} ^{q k+1}}^{(q(n-k))} \mathbb{1}\left(\left.\omega\right|_{q k} \tau, y\right) \\
& \quad+G_{j, \omega}^{n} \mathbb{1}(\tau, x) \\
& \leq \sum_{k=1}^{n} \sum_{V \in Z_{k}\left(z_{j}, \omega\right) \backslash W_{k}\left(z_{j}, \omega\right)} \sum_{y \in V \cap f_{\left.\omega\right|_{q k}}^{-1}(x)} \exp (q k \sup (\bar{\psi})) L_{\left.\omega\right|_{q n} ^{q k+1}}^{(q(n-k))} \mathbb{1}\left(\left.\omega\right|_{q k} \tau, y\right) \\
& \quad+\left\|G_{j, \omega}^{n} \mathbb{1}\right\|_{\infty} .
\end{aligned}
$$

Applying now Corollary $3.2\left(C_{k}\right)$ and $\left(D_{k}\right)$, we estimate further as follows.

$$
M_{j, \omega}^{n} \mathbb{1}(\tau, x) \leq \sum_{k=1}^{n} D\left(d q+\lambda^{-k}\right) \exp (q k \sup (\bar{\psi}))\left\|L_{\left.\omega\right|_{q n} ^{q k+1}}^{(q(n-k))} \mathbb{1}\right\|_{\infty}+\left\|G_{j, \omega}^{n} \mathbb{1}\right\|_{\infty} .
$$

Since $d q+\lambda^{-k} \leq \gamma_{q}^{-k}$ (see (4.5)), we thus get

$$
M_{j, \omega}^{n} \mathbb{1}(\tau, x) \leq D \sum_{k=1}^{n}\left(\exp (q \sup (\bar{\psi})) \gamma_{q}^{-1}\right)^{k}\left\|L_{\left.\omega\right|_{q n} ^{q k+1}}^{(q(n-k))} \mathbb{1}\right\|_{\infty}+\left\|G_{j, \omega}^{n} \mathbb{1}\right\|_{\infty} .
$$

Taking supremum over all $(\tau, x) \in \pi_{2}^{-1}\left(B\left(z_{j}, R_{q}\left(z_{j}\right)\right)\right)$, we thus get

$$
\left\|M_{j, \omega}^{n} \mathbb{1}\right\|_{\infty} \leq D \sum_{k=1}^{n}\left(\exp (q \sup (\bar{\psi})) \gamma_{q}^{-1}\right)^{k}\left\|L_{\left.\omega\right|_{q n} ^{q k+1}}^{(q(n-k))} \mathbb{1}\right\|_{\infty}+\left\|G_{j, \omega}^{n} \mathbb{1}\right\|_{\infty} .
$$


So, summing over all words $\in\{1, \ldots, s\}^{q n}$, we obtain using Lemma 4.3, the following.

$$
\begin{aligned}
\left\|\left|M_{j}^{n} \mathbb{1}\right|\right\|_{\infty} & \leq D \sum_{k=1}^{n}\left(\exp (q \sup (\bar{\psi})) \gamma_{q}^{-1}\right)^{k} s^{q k}\left|\left\|\mathcal{L}^{q(n-k)} \mathbb{1}\right\|\left\|_{\infty}+\right\|\right| G_{j}^{n} \mathbb{1} \mid \|_{\infty} \\
& \leq D \sum_{k=1}^{n} \exp (q k(\sup (\bar{\psi})+\log s))\left(\gamma_{q}^{-1}\right)^{k}\left\|\left|\mathcal{L}^{q(n-k)} \mathbb{1}\right|\right\|_{\infty}+Q_{\psi} \\
& =D \sum_{k=1}^{n}\left(\eta^{q} \gamma_{q}^{-1}\right)^{k}\|\| \mathcal{L}^{q(n-k)} \mathbb{1}|\||_{\infty}+Q_{\psi} .
\end{aligned}
$$

Since this inequality holds for all $j=1, \ldots, u$, we thus get

$$
\left\|\mathcal{L}^{q n} \mathbb{1}\right\|_{\infty} \leq D \sum_{k=1}^{n}\left(\eta^{q} \gamma_{q}^{-1}\right)^{k}\left\|\mid \mathcal{L}^{q(n-k)} \mathbb{1}\right\|_{\infty}+Q_{\psi} .
$$

The first inequality to be proved is thus established. In order to derive the second one from it, invoke (4.5).

Lemma 4.5. There exists a constant $\bar{Q}_{\psi}>0$ such that for all $n \geq 0$, we have

$$
\left\|\mathcal{L}^{n} \mathbb{1}\right\|_{\infty} \leq \bar{Q}_{\psi}
$$

Proof. We first shall prove by induction that

$$
\left\|\mid \mathcal{L}^{q n} \mathbb{1}\right\|_{\infty} \leq 2 Q_{\psi}
$$

for all integers $n \geq 0$. Since $\|\mathbb{1}\|_{\infty}=1$, this formula holds for $n=0$. So, fix $n \geq 1$ and suppose that (4.14) is true for all $0 \leq k \leq n-1$. It then follows from Lemma 4.4 that

$$
\begin{aligned}
\left\|\mid \mathcal{L}^{q n} \mathbb{1}\right\|_{\infty} & \leq Q_{\psi}+D \sum_{k=1}^{n}(1+2 D)^{-k} 2 Q_{\psi}=Q_{\psi}\left(1+2 D \sum_{k=1}^{n}(1+2 D)^{-k}\right) \\
& \leq 2 Q_{\psi},
\end{aligned}
$$

and (4.14) is proved. Since $\left\|\left|\mathcal{L}^{i+j} \mathbb{1}\right|\right\|_{\infty} \leq\left.\left\|\left|\mathcal{L}^{i} \mathbb{1}\right|\right\|\right|_{\infty}\left|\left\|\mathcal{L}^{j} \mathbb{1} \mid\right\|_{\infty}\right.$, we are done by setting $\bar{Q}_{\psi}=2 Q_{\psi} \max \left\{\left\||| \mathcal{L}^{k} \mathbb{1}\right\|_{\infty}: k=0,1, \ldots, q-1\right\}$.

Lemma 4.6. There exists a constant $\underline{Q}_{\psi}>0$ such that for all $n \geq 0$, we have

$$
\inf _{y \in J(f)} \mathcal{L}^{n} \mathbb{1}(y) \geq \underline{Q}_{\psi} .
$$


Proof. Taking $q \geq 1$ sufficiently large, we can make the product $\eta^{q} \gamma_{q}^{-1}$ (see (4.5)) as small as we wish. It therefore follows from (4.13) and Lemma 4.5 that for $q \geq 1$ large enough, for all $n \geq 0$ and all $1 \leq j \leq u$, we have

$$
\left\|\left|M_{j}^{n} \mathbb{1}\left\|_{\infty} \leq \frac{1}{2}+\right\|\right| G_{j}^{n} \mathbb{1} \mid\right\|_{\infty} .
$$

Since, by Lemma 4.1, $\int \mathcal{L}^{q n} \mathbb{1} d m_{\psi}=\int \mathbb{1} d m_{\psi}=1$, there thus exists $x \in J(f)$ such that $\mathcal{L}^{q n} \mathbb{1}(x) \geq 1$. Since $\left\{\pi_{2}^{-1}\left(B\left(z_{j}, R_{q}\left(z_{j}\right)\right)\right)\right\}_{j=1}^{u}$ is a cover of $J(f)$, there exists $1 \leq i \leq u$ such that $x \in \pi_{2}^{-1}\left(B\left(z_{i}, R_{q}\left(z_{i}\right)\right)\right)$. It follows from (4.15) that $\left\|\left|G_{i}^{n}(\mathbb{1})\right|\right\|_{\infty} \geq 1 / 2$. Applying now (4.9) we see that

$$
G_{i}^{n} \mathbb{1}(w) \geq\left(2 e^{2 H}\right)^{-1}
$$

for all $w \in \pi_{2}^{-1}\left(B\left(z_{i}, R_{q}\left(z_{i}\right)\right)\right)$. Take now an arbitrary point $y \in J(f)$. With $k \geq 1$, as in the proof of Lemma 4.3, it follows from (4.10) that there exists $\bar{y} \in \pi_{2}^{-1}\left(B\left(z_{i}, R_{q}\left(z_{i}\right)\right)\right)$ such that $f^{k q}(\bar{y})=y$. So, using (4.16) and the definition of the Perron-Frobenius operator, we obtain

$$
\begin{aligned}
\mathcal{L}^{q(n+k)} \mathbb{1}(y) & \geq \exp \left(S_{k q}(\bar{\psi}(\bar{y}))\right) \mathcal{L}^{q n} \mathbb{1}(\bar{y}) \geq \exp \left(\inf \left(S_{k q}(\bar{\psi})\right)\right) G_{i}^{n} \mathbb{1}(\bar{y}) \\
& \geq\left(2 e^{2 H}\right)^{-1} \exp \left(\inf \left(S_{k q}(\bar{\psi})\right)\right)
\end{aligned}
$$

Since $\inf \left(\mathcal{L}^{a+b} \mathbb{1}\right) \geq \inf \left(\mathcal{L}^{a} \mathbb{1}\right) \inf \left(\mathcal{L}^{b} \mathbb{1}\right)$, we are therefore done by taking $\underline{Q}_{\psi}:=$

$$
\min \left\{\left(2 e^{2 H}\right)^{-1} \exp \left(\inf \left(S_{k q}(\bar{\psi})\right)\right), 1\right\} \cdot \min \left\{\inf \left(\mathcal{L}^{u} \mathbb{1}\right): u=0,1, \ldots, k q-1\right\} .
$$

Now repeating verbatim the proof of Lemma 20 from [6], using Lemma 4.5 and Lemma 4.6, we get the following.

Lemma 4.7. Let $\tilde{h}(x):=\liminf _{n \rightarrow \infty} \frac{1}{n} \mathcal{L}^{n} \mathbb{1}(x), h_{1}(x):=\inf _{n \geq 0} \mathcal{L}^{n} \tilde{h}(x)$ and $h(x):=\frac{h_{1}(x)}{\int_{J(f)} h_{1} d m_{\psi}}$ for each $x \in J(f)$. Then $h: J(f) \rightarrow \mathbb{R}$ is a Borel measurable function such that the following hold.

(a)

$$
\frac{\underline{Q}_{\psi}}{\overline{\bar{Q}}_{\psi}} \leq h(x) \leq \frac{\bar{Q}_{\psi}}{\underline{Q}_{\psi}} \text { for every } x \in J(f) .
$$

(b) $\mathcal{L} h(x)=h(x)$ for every $x \in J(f)$.

(c) $\int h d m_{\psi}=1$. 
Remark 4.8. Note that up to a normalized factor (to make (c) hold) the function $h$ is independent of the conformal measure $m_{\psi}$.

As an immediate consequence of this lemma and Proposition 2.2 in [5], we have the following.

Theorem 4.9. The Borel probability measure $\mu_{\psi}=h m_{\psi}$ is f-invariant, equivalent to $m_{\psi}$, and $\frac{d \mu_{\psi}}{d m_{\psi}} \in\left[\underline{Q}_{\psi} / \bar{Q}_{\psi}, \bar{Q}_{\psi} / \underline{Q}_{\psi}\right]$.

\section{Equilibrium States}

Our objective in this section is to show that the measure $\mu_{\psi}$ produced in Theorem 4.9 is a unique equilibrium state for the potential $\psi$ and that it is ergodic. Let $\mathrm{P}(\psi)$ be the ordinary topological pressure of the potential $\psi$. If $\mu$ is a Borel probability $f$-invariant measure on $J(f)$, denote by $J_{\mu}$ its Jacobian with respect to the map $f$ (see page 108 in [10]). We start with the following.

Lemma 5.1. $\mathrm{P}(\psi) \geq \mathrm{P}_{b}(\psi)$.

Proof. Since $h_{\mu_{\psi}}(f) \geq \int_{J(f)} \log J_{\mu_{\psi}} d \mu_{\psi}$ (this is true for every finiteto-one endomorphisms and every probability invariant measure. See Lemma 10.5 and Theorem 5.14 in [10]) and since $J_{\mu_{\psi}}=\frac{h \circ f}{h} \exp \left(\mathrm{P}_{b}(\psi)-\right.$ $\psi$ ) everywhere, it follows from Theorem 4.9 and the Variational Principle that

$$
\mathrm{P}(\psi) \geq h_{\mu_{\psi}}(f)+\int \psi d \mu_{\psi} \geq \int\left(\mathrm{P}_{b}(\psi)-\psi+\psi\right) d \mu_{\psi}=\mathrm{P}_{b}(\psi) .
$$

We are done.

Now, let $\mu$ be a Borel $f$-invariant ergodic measure on $J(f)$ such that $h_{\mu}(f \mid \sigma)>0$, where $h_{\mu}(f \mid \sigma)$ denotes the relative entropy of $(f, \mu)$ with respect to $\sigma$, and let $T_{\mu}: L_{\infty}(\mu) \rightarrow L_{\infty}(\mu)$ be the Perron-Frobenius operator associated to the measure $\mu$. It is defined by the formula

$$
T_{\mu} g(x)=\sum_{y \in f^{-1}(x)} J_{\mu}^{-1}(y) g(y) .
$$


Since $\mathcal{L} h=h$ everywhere throughout $J(f)$, using Lemma 6.9 from [13], we get that

$$
\begin{aligned}
1 & =\int \mathbb{1} d \mu=\int \frac{\mathcal{L} h}{h} d \mu=\int T_{\mu}\left(\frac{h \exp (\bar{\psi})}{J_{\mu}^{-1} \cdot h \circ f}\right) d \mu=\int \frac{h \exp (\bar{\psi})}{J_{\mu}^{-1} \cdot h \circ f} d \mu \\
& \geq 1+\int \log \left(\frac{h \exp (\bar{\psi})}{J_{\mu}^{-1} \cdot h \circ f}\right) d \mu \\
& =1+\int \log h d \mu-\int \log (h \circ f) d \mu+\int \bar{\psi} d \mu+\int \log J_{\mu} d \mu \\
& =1+\int \psi d \mu-\mathrm{P}_{b}(\psi)+\mathrm{h}_{\mu}(f) .
\end{aligned}
$$

Seeking contradiction suppose that $\mathrm{P}(\psi)>\mathrm{P}_{b}(\psi)$. By the Variational Principle there exists a Borel probability $f$-invariant ergodic measure $\mu$ such that

$$
\mathrm{h}_{\mu}(f)+\int \psi d \mu>\mathrm{P}(\psi)-\min \left\{\mathrm{P}(\psi)-\mathrm{P}_{b}(\psi), \mathrm{P}(\psi)-\sup (\psi)-\log s\right\} .
$$

Note that, because of (4.3), $\mathrm{P}(\psi)-\sup (\psi)-\log s>0$, and therefore

$$
\begin{aligned}
\mathrm{h}_{\mu}(f) & >\mathrm{P}(\psi)-(\mathrm{P}(\psi)-\sup (\psi)-\log s)-\int \psi d \mu \\
& =\log s+\sup (\psi)-\int \psi d \mu \\
& \geq \log s,
\end{aligned}
$$

which implies $h_{\mu}(f \mid \sigma)>0$. Hence, (5.3) applies, and we can continue it to get that $1 \geq 1+\int \psi d \mu-\mathrm{P}_{b}(\psi)+\mathrm{h}_{\mu}(f)>1+\mathrm{P}(\psi)-\left(\mathrm{P}(\psi)-\mathrm{P}_{b}(\psi)\right)-$ $\mathrm{P}_{b}(\psi)=1$. This contradiction along with (5.1) and Lemma 5.1 give the following.

Proposition 5.2. $\mathrm{P}(\psi)=\mathrm{P}_{b}(\psi)$ and $\mu_{\psi}$ is an equilibrium state for $\psi$.

Lemma 5.3. For each point $x \in J(f)$, we have $\frac{1}{n} \log \mathcal{L}_{\psi}^{n} \mathbb{1}(x) \rightarrow \mathrm{P}(\psi)$ as $n \rightarrow \infty$.

Proof. By Proposition 5.2, $\mathcal{L}_{\psi}=(\exp (\mathrm{P}(\psi))) \cdot \mathcal{L}$. Combining it with Lemma 4.5 and Lemma 4.6, we obtain that the statement of our lemma holds.

Now suppose that $\mu$ is an arbitrary ergodic equilibrium state for $\psi$. Then $h_{\mu}(f)-\log s=\mathrm{P}(\psi)-\int \psi d \mu-\log s \geq \mathrm{P}(\psi)-\sup (\psi)-\log s>0$. Hence $h_{\mu}(f \mid \sigma)>0$. In view of the proposition above, the last component in (5.3) is equal to 1 . Consequently, the only inequality in this formula 
becomes an equality, and so $\log \left(\frac{h \exp (\bar{\psi})}{J_{\mu}^{-1} \cdot h \circ f}\right)=0 \mu$-a.e. We thus get the following.

Lemma 5.4. If $\mu$ is an ergodic arbitrary equilibrium state for $\psi$, then $J_{\mu}=\frac{h \circ f}{h} \exp (\mathrm{P}(\psi)-\psi) \mu$-a.e.

Now we shall prove the following.

Lemma 5.5. If a Borel $f$-invariant probability measure $\mu$ satisfies $J_{\mu}=$ $\frac{h \circ f}{h} \exp (\mathrm{P}(\psi)-\psi) \mu$-a.e., and if a Borel $f$-invariant probability measure $m$ satisfies $J_{m}=\frac{h \circ f}{h} \exp (\mathrm{P}(\psi)-\psi)$ everywhere, then $\mu$ is absolutely continuous with respect to $\mathrm{m}$.

Proof. It suffices to prove that for every $\varepsilon>0$ there exists $\delta>0$ such that if $g: J(f) \rightarrow(0,1]$ is a continuous function and $\int g d m \leq \delta$, then $\int g d \mu \leq \varepsilon$. Taking $q=q(\epsilon) \geq 1$ large enough, taking points $z_{j}$ $(j=1, \ldots, u)$ in $J(G)$ such that $\cup_{j=1}^{u} B\left(z_{j}, R_{q}\left(z_{j}\right)\right) \supset J(G)$, using (4.5), Lemma 4.5, and redoing the considerations between (4.12) and (4.13) with the function $\mathbb{1}$ replaced by the function $g$, and $\left\|G_{j, \omega}^{n} \mathbb{1}\right\|_{\infty}$ replaced by $G_{j, \omega}^{n} g(\tau, x)$, after applying (5.2), we get for every $1 \leq j \leq u$, every $n=l \cdot q \geq 1(l \in \mathbb{N})$ and every $x \in \pi_{2}^{-1}\left(B\left(z_{j}, R_{q}\left(z_{j}\right)\right)\right)$, that

$$
T_{\mu}^{q n} g(x) \leq \frac{\varepsilon}{2}+C G_{j}^{n} g(x) \text { and } T_{m}^{q n} g(x) \geq C^{-1} G_{j}^{n} g(x),
$$

where $C=\bar{Q}_{\psi} / \underline{Q}_{\psi}$ is a positive constant which does not depend on $l, j, x, g$. We take a partition of the set $J(G)$ into mutually disjoint sets $\left\{Y_{j}^{i}: 1 \leq j \leq u, 1 \leq i \leq i(j)\right\}$ each of which has a positive $m \circ \pi_{2}^{-1}$ measure and $\bigcup_{i=1}^{i(j)} Y_{j}^{i} \subset B\left(z_{j}, R_{q}\left(z_{j}\right)\right)$ for all $j=1,2, \ldots, u$. (Note that we can take such a partition, since the support of $m$ is equal to $J(f)$, which follows from the topological exactness of $f: J(f) \rightarrow J(f)$ and the condition " $J_{m}=\frac{h \circ f}{h} \exp (\mathrm{P}(\psi)-\psi)$ everywhere".) Put

$$
\alpha=\sup \left\{\frac{\mu \circ \pi_{2}^{-1}\left(Y_{j}^{i}\right)}{m \circ \pi_{2}^{-1}\left(Y_{j}^{i}\right)}: 1 \leq j \leq u, 1 \leq i \leq i(j)\right\} .
$$

Since $g$ is everywhere positive, there exist $\zeta>0$ and $k \geq 1$ such that

$$
\frac{1}{2} \leq \frac{g(\theta, y)}{g(\rho, z)} \leq 2
$$


for all $(\theta, y),(\rho, z) \in J(f)$ with $d(y, z)<\zeta$ and $\left.\theta\right|_{k}=\left.\rho\right|_{k}$. Fix now $n=$ $l \cdot q \geq 1$ so large that for each $1 \leq j \leq u, K_{G}\left(R_{q}^{\prime}\left(z_{j}\right)\right)^{4} \kappa^{-1} \lambda^{n / 2} C_{s a}^{1 / 2}<\zeta$ (see item $\left(B_{n}\right)$ of Corollary 3.2). Using then item $\left(B_{n}\right)$ of this corollary and (5.5), the application of (4.7) gives for all $n=l \cdot q \geq k$, all $\omega \in$ $\{1, \ldots, s\}^{n}$, and all $\xi, x \in \pi_{2}^{-1}\left(B\left(z_{j}, R_{q}\left(z_{j}\right)\right)\right)$, that

$$
\frac{1}{2} e^{-2 H} G_{j, \omega}^{n} g(x) \leq G_{j, \omega}^{n} g(\xi) \leq 2 e^{2 H} G_{j, \omega}^{n} g(x) .
$$

Hence summing over all $\omega \in\{1, \ldots, s\}^{n}$, we get

$$
\frac{1}{2} e^{-2 H} G_{j}^{n} g(x) \leq G_{j}^{n} g(\xi) \leq 2 e^{2 H} G_{j}^{n} g(x)
$$

for all $n=l \cdot q \geq k$, all $1 \leq j \leq u$ and all $\xi, x \in \pi_{2}^{-1}\left(B\left(z_{j}, R_{q}\left(z_{j}\right)\right)\right)$. Let $X_{j}^{i}:=\pi_{2}^{-1}\left(Y_{j}^{i}\right)$. Now applying (5.4), (5.6), and the definition of $\alpha$, we get

$$
\begin{aligned}
\int g d \mu & =\int T_{\mu}^{q n} g d \mu \leq \frac{\varepsilon}{2}+\sum_{j=1}^{u} C \sum_{i=1}^{i(j)} \int_{X_{j}^{i}} G_{j}^{n} g d \mu \\
& \leq \frac{\varepsilon}{2}+\sum_{j=1}^{u} C \sum_{i=1}^{i(j)} \mu\left(X_{j}^{i}\right) \sup _{X_{j}^{i}}\left(G_{j}^{n} g\right) \\
& \leq \frac{\varepsilon}{2}+\sum_{j=1}^{u} C \sum_{i=1}^{i(j)} 2 e^{2 H} \mu\left(X_{j}^{i}\right) \inf _{X_{j}^{i}}\left(G_{j}^{n} g\right) \\
& \leq \frac{\varepsilon}{2}+2 e^{2 H} \sum_{j=1}^{u} C \sum_{i=1}^{i(j)} \frac{\mu\left(X_{j}^{i}\right)}{m\left(X_{j}^{i}\right)} \int_{X_{j}^{i}} G_{j}^{n} g d m \\
& \leq \frac{\varepsilon}{2}+2 e^{2 H} \alpha \sum_{j=1}^{u} C^{2} \sum_{i=1}^{i(j)} \int_{X_{j}^{i}} T_{m}^{q n} g d m \\
& =\frac{\varepsilon}{2}+2 e^{2 H} \alpha C^{2} \int T_{m}^{q n} g d m=\frac{\varepsilon}{2}+2 e^{2 H} \alpha C^{2} \int g d m .
\end{aligned}
$$

So, taking $\delta=\left(4 \alpha C^{2} e^{2 H}\right)^{-1} \varepsilon$, finishes the proof.

Lemma 5.6. If $\mu$ is an ergodic equilibrium state, and if a Borel $f$ invariant probability measure $m$ satisfies $J_{m}=\frac{h \circ f}{h} \exp (\mathrm{P}(\psi)-\psi)$ everywhere, then $\mu$ is absolutely continuous with respect to $m$.

Proof. By Lemma 5.4 and Lemma 5.5, we obtain the statement.

We conclude the paper with the following. 
Theorem 5.7. The measure $\mu_{\psi}$ forms a unique (ergodic) equilibrium state for $\psi$ and $m_{\psi}$ is a unique $\exp (P(\psi)-\psi)$-conformal measure.

Proof. For every $\exp (\mathrm{P}(\psi)-\psi)$-conformal measure $\nu$ let $h=h^{\nu}$ be the density function obtained in Lemma 4.7 with $m_{\psi}$ replaced by $\nu$. Note that by Remark 4.8, they differ by a positive multiplicative constant. Suppose that there exist two $\exp (\mathrm{P}(\psi)-\psi)$-conformal measures $m_{1}$ and $m_{2}$ that are not equivalent. Then there exists a Borel set $A \subset$ $J(f)$ such that $m_{1}(A)>0$ and $m_{2}(A)=0$. Hence, on the one hand, $h^{m_{1}} m_{1}(A)>0$ since, by Lemma $4.7, h^{m_{1}}>0$ everywhere, and, on the other hand, $h^{m_{1}} m_{1}(A)=0$ since each measure $\tau_{i}=h^{m_{i}} m_{i}(i=1,2)$ satisfies $J_{\tau_{i}}=\frac{h^{m_{i} \circ f}}{h^{m_{i}}} \exp (\mathrm{P}(\psi)-\psi)$ everywhere and hence these two measures are equivalent by Lemma 5.5. So, any two $\exp (\mathrm{P}(\psi)-\psi)$ conformal measures are equivalent. Now suppose that there are two different ergodic equilibrium states $\mu_{1}$ and $\mu_{2}$ for $\psi$. Then they are mutually singular and there is a completely invariant Borel set $A \subset J(f)$ $\left(f(A)=A=f^{-1}(A)\right)$ such that $\mu_{1}(A)=1$ and $\mu_{2}(A)=0$ (which implies that $\left.\mu_{2}(J(f) \backslash A)=1\right)$. Then, by Lemma $5.6, m_{\psi}(A)>0$ and $m_{\psi}(J(f) \backslash A)>0$. Hence, both Borel probability measures $m_{\psi}^{1}$ and $m_{\psi}^{2}$ on $J(f)$, respectively defined by the formulas

$$
m_{\psi}^{1}(F)=\frac{m_{\psi}(F \cap A)}{m_{\psi}(A)} \text { and } m_{\psi}^{2}(F)=\frac{m_{\psi}(F \cap(J(f) \backslash A))}{m_{\psi}(J(f) \backslash A)},
$$

are $\exp (\mathrm{P}(\psi)-\psi)$-conformal. Since they are singular, we get a contradiction, and the uniqueness of equilibrium state for $\psi: J(f) \rightarrow \mathbb{R}$ is established. So, coming back to conformal measures, if $\nu_{1}$ and $\nu_{2}$ are two $\exp (\mathrm{P}(\psi)-\psi)$-conformal measures, then $h^{\nu_{1}} \nu_{1}=h^{\nu_{2}} \nu_{2}$. Since the ratio $h^{\nu_{2}} / h^{\nu_{1}}$ is constant, so is the ratio $\nu_{2} / \nu_{1}$. Since, in addition, both these measures $\nu_{2}$ and $\nu_{1}$ are probabilistic, they are equal. We are done.

Proof of the main result Theorem 1.1: Combining Theorem 5.7 and Lemma 5.3, the statement of the theorem follows.

\section{REFERENCES}

[1] R. Brück, Geometric properties of Julia sets of the composition of polynomials of the form $z^{2}+c_{n}$, Pacific J. Math., 198 (2001), no. 2, 347-372.

[2] M. Büger, Self-similarity of Julia sets of the composition of polynomials, Ergod. Th. and Dynam. Sys. 17 (1997), 1289-1297.

[3] M. Büger, On the composition of polynomials of the form $z^{2}+c_{n}$, Math. Ann. 310 (1998), no. 4, 661-683. 
[4] R. Brück, M. Büger and S. Reitz, Random iterations of polynomials of the form $z^{2}+c_{n}$ : Connectedness of Julia sets, Ergod. Th. and Dynam. Sys., 19, (1999), No.5, 1221-1231.

[5] M. Denker, M. Urbański, On the existence of conformal measures, Trans. A.M.S. 328 (1991), 563-587.

[6] M. Denker, M. Urbański, Ergodic theory of equilibrium states for rational maps, Nonlinearity 4 (1991), 103-134.

[7] J. E. Fornaess and N. Sibony, Random iterations of rational functions, Ergod. Th. and Dynam. Sys. 11(1991), 687-708.

[8] A. Hinkkanen and G.J.Martin, The Dynamics of Semigroups of Rational Functions I, Proc. London Math. Soc. (3)73(1996), 358-384.

[9] M. Lyubich, Entropy properties of rational endomorphisms of the Riemann sphere. Ergod. Th. Dynam. Sys. 3 (1983), 351-386.

[10] W. Parry, Entropy And Generators In Ergodic Theory, Mathematics Lecture Note Series, 1969, Benjamin Inc.

[11] D. Ruelle, Thermodynamic formalism, Encyclopedia of Math. and Appl., vol. 5, Addison - Wesley, Reading Mass., 1976.

[12] R. Stankewitz and H. Sumi, Dynamical properties and structure of Julia sets of postcritically bounded polynomial semigroups, preprint, http://arxiv.org/abs/0708.3187

[13] H. Sumi, Skew product maps related to finitely generated rational semigroups, Nonlinearity 13 (2000), 995-1019.

[14] H. Sumi, Dynamics of sub-hyperbolic and semi-hyperbolic rational semigroups and skew products, Ergod. Th. and Dynam. Sys.(2001), 21, 563603.

[15] H. Sumi, Dimensions of Julia sets of expanding rational semigroups, Kodai Mathematical Journal, 28, No.2, 2005, pp390-422.

[16] H. Sumi, Semi-hyperbolic fibered rational maps and rational semigroups, Ergod. Th. and Dynam. Sys. (2006), 26, 893-922.

[17] H. Sumi, Random dynamics of polynomials and devil's-staircase-like functions in the complex plane, Appl. Math. Comput. 187 (2007), no. 1, 489500. (Proceedings paper.)

[18] H. Sumi, Dynamics of postcritically bounded polynomial semigroups, preprint 2007, http://arxiv.org/abs/math/0703591.

[19] H. Sumi and M. Urbański, Real analyticity of Hausdorff dimension for expanding rational semigroups, preprint 2007, http://arxiv.org/abs/0707.2447.

[20] P. Walters, An Introduction To Ergodic Theory, Springer-Verlag, 1982.

[21] W. Zhou, F.Ren, The Julia sets of the random iteration of rational functions, Chinese Bulletin, 37(12), 1992, 969-971.

Hiroki Sumi; Department of Mathematics, Graduate School of Science, Osaka University, 1-1 Machikaneyama, Toyonaka, Osaka, 5600043, JAPAN

sumi@math.sci.osaka-u.ac.jp, http://www.math.sci.osaka-u.ac.jp/ sumi/ 
THE EQUILIBRIUM STATES FOR SEMIGROUPS OF RATIONAL MAPS 23

Mariusz Urbański; Department of Mathematics, University of North Texas, P.O. Box 311430, Denton TX 76203-1430, USA

urbanski@unt.edu, http://www.math.unt.edu/ urbanski 